\title{
Editorial
}
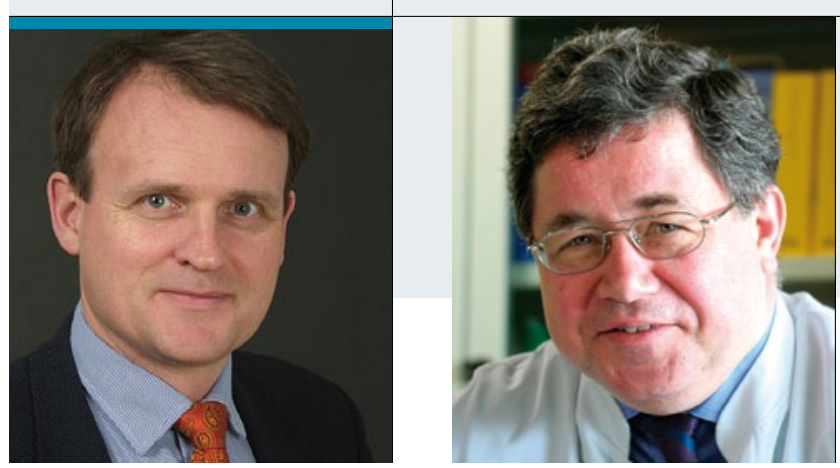

"Der Schritt von der Extrakt-basierten zur

Einzelallergen-basierten Diagnostik ist aus der

Allergologie gar nicht mehr wegzudenken."

Prof. Dr. Thilo Jakob, Allergieabteilung \& Forschergruppe

Allergologie, Klinik für Dermatologie \& Venerologie

Universitätsklinikum, Freiburg

Prof. Dr. Hans F. Merk, Hautklinik der Medizinischen

Fakultät, Universitätsklinikum der RWTH Aachen

\section{Paralyse durch Analyse?}

$\mathrm{E}$ nde Juni trafen sich in Mailand knapp 8.000 Allergologen zum EAACI-WAO-Allergie-Weltkongress. Neben vielen anderen Themen zog sich die molekulare Allergiediagnostik durch fast alle Sitzungen. Über 30 Jahre liegt es zurück, dass die ersten Einzelallergene kloniert wurden und als rekombinante Proteine für diagnostische Zwecke zur Verfügung standen. Aber erst in den letzten fünf Jahren ist so richtig Bewegung in das Gebiet gekommen unterdessen können weit über 100 Einzelallergene für die Allergiediagnostik im klinischen Alltag eingesetzt werden. Langsam, aber sicher findet hier ein Umdenken statt. Während die primäre Diagnostik häufig noch mit Allergenextrakten durchgeführt wird, ermöglicht die Verfügbarkeit von Markerallergenen eine verbesserte Abgrenzung zwischen genuiner Sensibilisierung und Kreuzsensibilisierung bei polysensibilisierten Patienten. Zudem lassen sich v.a. im Bereich der Nahrungsmittelallergie Sensibilisierungen gegenüber Risikoallergenen identifizieren.

Der Schritt von der Extrakt-basierten zur Einzelallergen-basierten Diagnostik ist aus vielen Bereichen der Allergologie gar nicht mehr wegzudenken. Die hierdurch erreichte Verbesserung der Auflösung und Präzision scheint manchen Kollegen aber noch nicht auszureichen: Tatsächlich gibt es Hinweise darauf, dass die Analyse der IgE-Reaktivität auf der Ebene einzelner Epitope eine weitere Präzision in die Diagnostik bringen könnte. So zeigen Patienten mit hochaffinen IgE-Antikörpern, die eine Vielzahl von sequenziellen Epitopen erkennen, schwerere allergische Reaktionen und reagieren auf geringere Dosen während der Nahrungsmittelprovokation als Patienten, die wenige IgE-Epitope mit niedriger Affinität erkennen. Zusätzlich sind bestimmte Profile mit einer erhöhten Persistenz der Nahrungsmittelallergie assoziiert. Ob sich die von der Arbeitsgruppe von Hugh Sampson, New York, vorgestellten Daten auch auf andere Allergene übertragen lassen, werden zukünftige Studien zeigen müssen.

Wenn wir eine ähnliche Entwicklung wie im Bereich der Humangenetik prognostizieren, wäre es denkbar, dass wir in einigen Jahren in der Lage sind das komplette "Sensitis-ome“ eines Patienten auf Epitopebene („Epit-ome“) abzubilden. Dann hätten wir eine ähnliche Situation wie in der Humangenetik, wo es bald möglich ist, das gesamte Genom eines Patienten für ein paar tausend Euro zu sequenzieren, es dann aber mehrere $100.000 €$ kostet, die verfügbare Information richtig zu interpretieren. Um nicht auch in der Allergologie in eine derartige „Paralyse durch Analyse" (frei nach Steve Taylor, Nebraska, USA) zu geraten, müssen intelligente Programme entwickelt werden, die besonders bei Multiplexanalysen dem Nutzer Interpretationshilfen und Hintergrundinformationen liefern. Hier besteht aktuell noch großer Entwicklungsbedarf.

Ähnlich komplex wie die o.g. Materie scheint die Abrechnungssituation für SCIT oder SLIT zu sein. Während die Hyposensibilisierung durch subkutane Injektionen durch eine separate Gebührenordnungsposition über die Abrechnung nachweisbar ist, trifft dies nicht für die sublinguale Therapie zu. Wenn Sie überdurchschnittlich viele Patienten hyposensibilisieren, kann dies zum Überschreiten ihres Richtgrößenbudgets führen und ggf. zur entsprechenden Überprüfung. Die unterschiedliche Handhabe der SCIT und SLIT hat die kassenärztliche Vereinigung Hessen dazu veranlasst, eine Pseudogebührenordnungsposition 98520 „Hyposensibilisierungsbehandlung durch subkutane Injektionen oder orale Therapeutika" einzuführen, mit der Sie die Möglichkeit haben in einem evtl. Prüfverfahren auch Leistungen der SLIT als mögliche Praxisbesonderheit geltend zu machen.

In der aktuellen Ausgabe bieten wir Ihnen wieder einen Beitrag aus der Serie molekulare Allergiediagnostik zum Nutzen bei nahrungsmittelabhängiger anstrengungsinduzierter Anaphylaxie (S. 308), zwei Übersichtsbeiträge zum Thema der oralen Toleranz (S. 312, S. 317) und abschließend einen hoch aktuellen CME-Beitrag zur zeitgemäßen Diagnostik und Therapie der chronisch spontanen Urtikaria (S. 324).
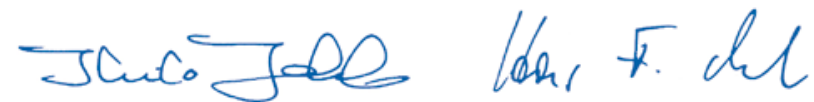